\title{
REPORTE DE UN CASO
}

Revista Facultad de Salud - RFS - Enero - Junio 2012;4-1:99-106

\section{SÍNDROME DE EISENMENGER Eisenmenger Syndrome}

\author{
Juan Pablo González Suárez¹, Giovanni Caviedes Pérez²
}

Recibido: 11 de abril de 2012 - Aceptado: 18 de junio de 2012

${ }^{1}$ Estudiante de medicina. Facultad de Salud, Universidad Surcolombiana. Correo electrónico: juanpagonzalez07@hotmail.com

${ }^{2}$ Médico internista. Farmacólogo clínico, Epidemiólogo. Docente, Universidad Surcolombiana. Correo electrónico: giocape@hotmail.com

\section{Resumen}

La cardiopatía congénita acianótica frecuentemente diagnosticada en el adulto es la comunicación interauricular. Se presenta un reporte de caso sobre un paciente de 27 años con diagnóstico de aurícula única con leve sintomatología a pesar de la edad y sin realizarse una corrección quirúrgica; actualmente se encuentra en buen estado general, algo inesperado para la edad.

Palabras clave: Comunicación interauricular, Sindrome de Eisenmenger.

\begin{abstract}
Acyanotic congenital heart disease frequently diagnosed in adults is the atrial septal defect. It is a case report of a 27-year-old patient diagnosed with unique atrium with mild symptoms despite his age and without undergoing a correction surgery; currently the patient is in good general condition, something unexpected for his age.
\end{abstract}

Key words: Atrial septal defect, Eisenmenger Syndrome.

\section{INTRODUCCIÓN}

De las cardiopatías congénitas más frecuentes, la comunicación interauricular, corresponde a un $30 \%$ a $40 \%$ de las cardiopatías más comunes en los adultos, con una prevalencia de 2,8 a 8,8 por cada 1.000 nacidos vivos y una relación de 1:2 entre hombres y mujeres respectivamente. La asociación de las cardiopatías congénitas, con enfermedades vasculares pulmonares tiene una incidencia de 8 a 12 por cada 1.000 nacimientos; y una prevalencia cercana al $60 \%$ de todas las cardiopatías congénitas principalmente en niños escolares ${ }^{(1,2)}$.

\section{REPORTE DE CASO}

Paciente masculino de 27 años con cuadro de diez días de evolución consistente en dolor torácico, tipo opresivo, de aparición súbita, intensidad de 3/10, irradiado a espalda, que mejora con la marcha lenta y empeora con el decúbito supino asociado a disnea de leves esfuerzos, ortopnea y palpitaciones. Tiene un antecedente de trauma facial en la niñez y trae la imagen de un ecocardiograma realizado en una clínica de Bogotá (Figura 1) donde muestra la aparición de una cavidad auricular única. $\mathrm{Al}$ examen físico se encuentra una $\mathrm{TA}=80 / 50 \mathrm{mmHg}, \mathrm{FC}=64 \mathrm{LPM}, \mathrm{FR}=24 \mathrm{RPM}$, 
$\mathrm{T}^{\circ} \mathrm{ax}=35^{\circ} \mathrm{C}$; depresión hemicraneana derecha, cianosis labial, tórax en tonel (Figura 2) con una expansibilidad disminuida y reforzamiento del $2^{\circ}$ ruido cardíaco con presencia de un soplo sistólico tricúspideo (soplo en maquinaria), abdomen normal y extremidades superiores e inferiores con hipocratismo digital (Figura 3). El test neurológico es normal. Se realiza ecocardiograma descrito en la Tabla 1.

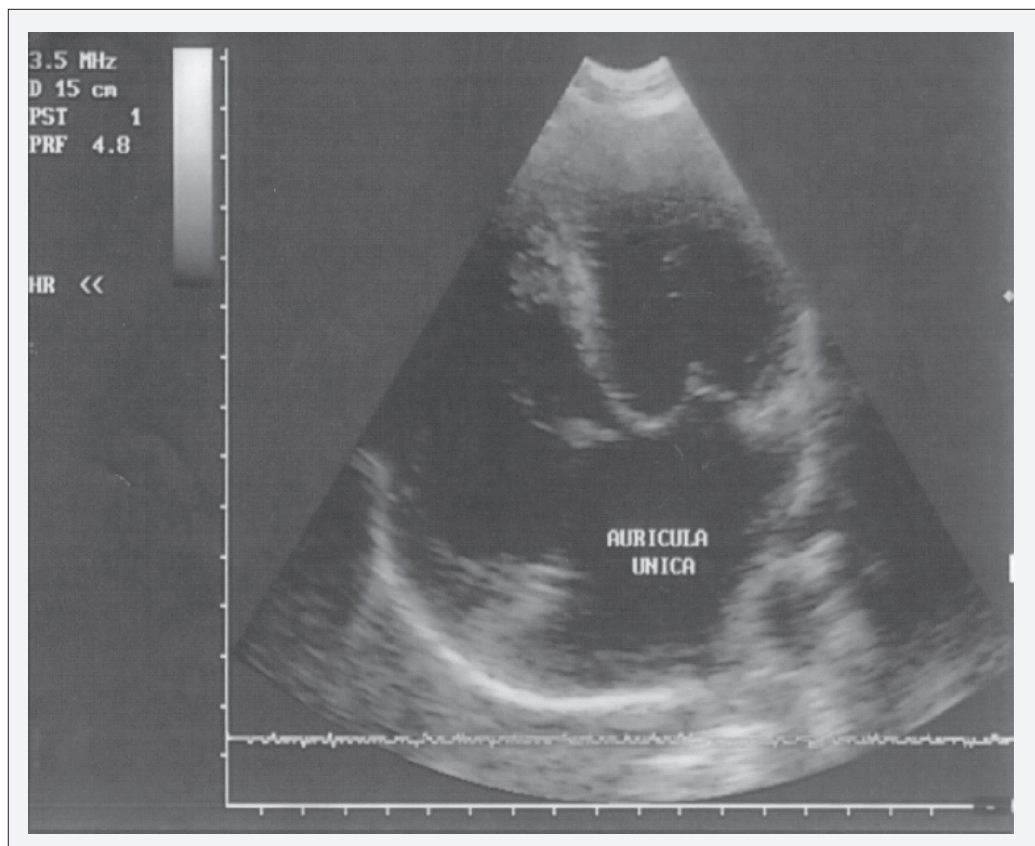

Figura 1. Ecocardiograma realizado a los 17 años.

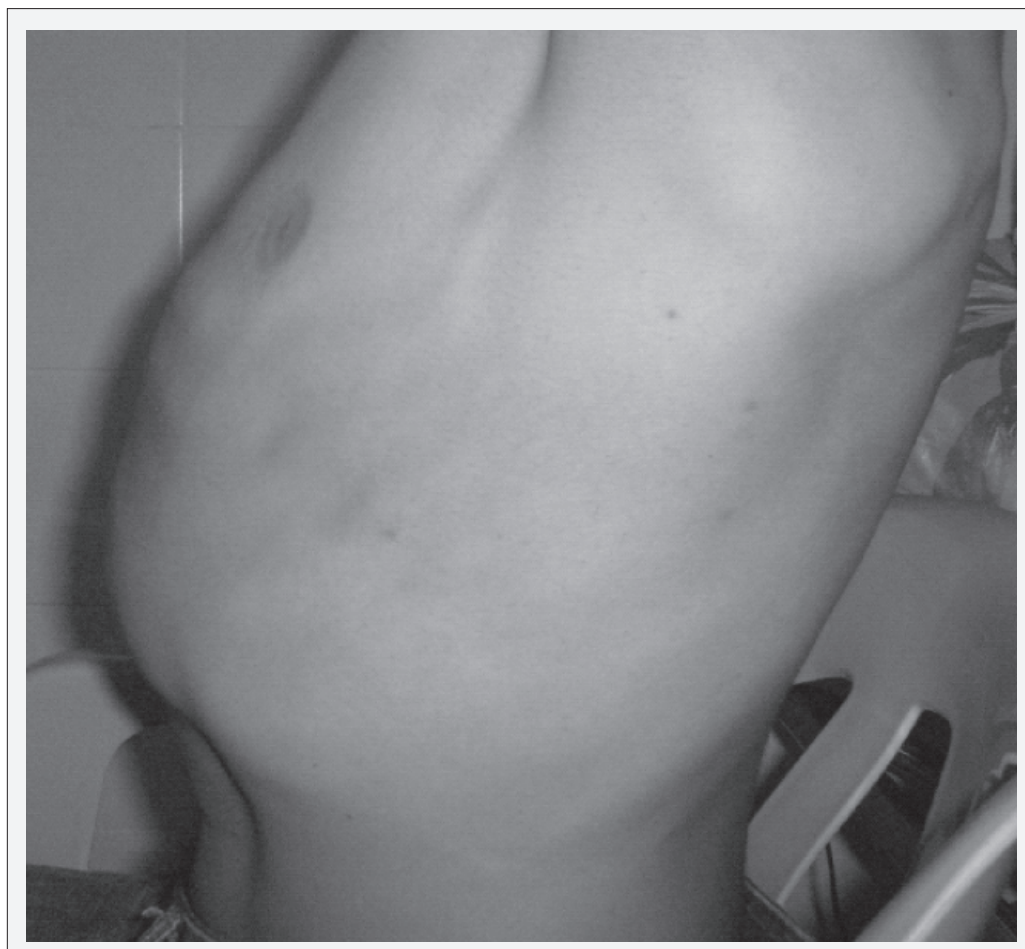

Figura 2. Tórax en Tonel. 
SÍNDROME DE EISENMENGER

Tabla 1. Cuadro comparativo entre los resultados de los ecocardiogramas realizados en un hospital de tercer nivel y el Hospital Universitario Hernando Moncaleano Perdomo.

\begin{tabular}{|c|c|c|}
\hline Descripción & Primer Hospital (17 años) & H.U.H.M.P (27 años) \\
\hline & $\begin{array}{l}\text { - Tabique interauricular ausente. } \\
\text { - } \text { Doble sistema de venas cavas superiores. } \\
\text { - Conexiones auriculo-ventriculares: } \\
\text { aurícula única conectada a través de dos } \\
\text { válvulas. } \\
\text { - } \quad \text { Tabique interventricular: soluciones de con- } \\
\text { tinuidad a nivel musculares apicales múl- } \\
\text { tiples siendo la más grande de ella de } 6 \text { mm } \\
\text { de diámetro no pudiéndose constatar cor- } \\
\text { tocircuito por estar igualadas las presiones. } \\
\text { - Ventrículo derecho severamente hipertrófico } \\
\text { con signos de sobrecarga de presión. } \\
\text { - Insuficiencia tricuspidea leve. } \\
\text { - Arteria pulmonar principal y ramas seve- } \\
\text { ramente dilatadas. }\end{array}$ & $\begin{array}{l}\text { - } \quad \text { Aurícula única. } \\
\text { - } \text { Insuficiencia tricuspidea leve. } \\
\text { - } \text { Aorta bivalva. } \\
\text { - Ventrículo derecho: espesor aumen- } \\
\text { tado de su pared. } \\
\text { - } \quad \text { Arteria pulmonar: tronco y ramas de } \\
\text { características normales. } \\
\text { - Tabique interventricular: integro. } \\
\text { - Tabique interauricular: esbozo de } \\
\text { septum interauricular inferior. }\end{array}$ \\
\hline Conclusiones & $\begin{array}{l}\text { 1. Aurícula única } \\
\text { 2. Comunicación interventricular musculares } \\
\text { apicales múltiples. } \\
\text { 3. Hipertensión pulmonar severa. } \\
\text { 4. Doble sistema de vena cava superior. } \\
\text { 5. Aorta Bivalva. } \\
\text { 6. Arterias pulmonares severamente dilatadas. } \\
\text { 7. Ventrículo derecho severamente hiper- } \\
\text { trófico. } \\
\text { 8. Buena Función bi-ventricular. }\end{array}$ & $\begin{array}{l}\text { 1. Aurícula única con válvula mitral y } \\
\text { tricuspidea presentes y morfoló- } \\
\text { gicamente normales. } \\
\text { 2. Engrosamiento importante de las pa- } \\
\text { redes del ventrículo derecho, por so- } \\
\text { brecarga sistólica crónica pulmonar. } \\
\text { 3. Aorta Bivalva. } \\
\text { 4. Insuficiencia Tricuspidea Leve. } \\
\text { 5. Presión arterial sistólica transpul- } \\
\text { monar de } 50 \text { mmHg. } \\
\text { 6. Cardiopatía congénita compleja. }\end{array}$ \\
\hline
\end{tabular}

Respecto a los paraclínicos, el electrocardiograma muestra un patrón de bloqueo de rama izquierda (Figura 4); cuadro hemático con policitemia (Tabla 2) y gases arteriales con hipoxemia (Tabla 3). Las pruebas de coagulación, función renal y la glicemia son normales (Tabla 4). Con estos resultados se establecen los siguientes diagnósticos: 1) Síndrome de Eisenmenger; 2) Hipertensión pulmonar; 3) Cardiopatía congénita; y 4) Síndrome disneico. El tratamiento que instaurado de urgencia consistió en dalteparina 5000 UI SC día, enalapril 5 mg VO día, furosemida $5 \mathrm{mg} \mathrm{IV} \mathrm{C} / 6 \mathrm{~h}$, lovastatina $40 \mathrm{mg}$ VO noche, Omeprazol 20 mg VO día, O2 por cánula 2L por minuto permanente $\mathrm{y}$ pulsoximetria $\mathrm{C} / 4 \mathrm{~h}$.

Tan pronto se estabiliza el paciente, se procede a explicar en qué consiste su enfermedad y se le aclara cuáles son los tratamientos indicados para dicho caso; de igual forma se le explican cuáles son las ventajas y desventajas de cada uno. Posteriormente, se le da el tiempo necesario para tomar su decisión que consistió en continuar con su tratamiento inicial.

Después de once días de observación se decide dar alta por mejoría de los síntomas. El paciente refiere realizar sus actividades diarias sin ningún problema y hace uso diario durante la noche del oxígeno. 

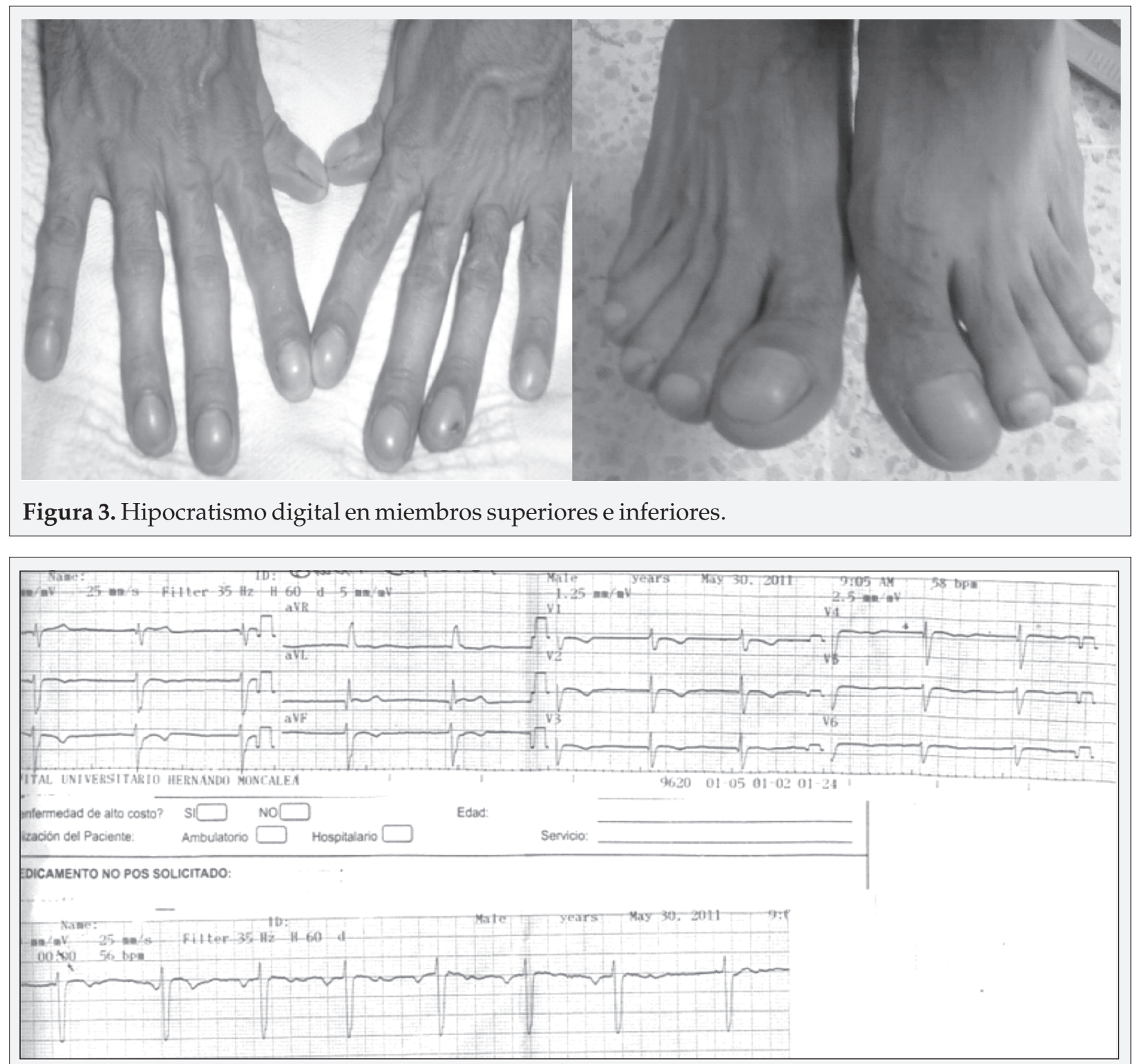

Figura 4. Electrocardiograma.

Tabla 2. Resultados del cuadro hemático.

Recuentos de Blancos
Neutrófilos
Linfocitos
Monocitos
Eosinófilos
Basófilos
Recuento de rojos
Hemoglobina
Hematocrito
VCM
RDW
Plaquetas

$6.900 \times \mathrm{mm}^{3}$
$58,5 \%$
$32,6 \%$
$6,9 \%$
$1,6 \%$
$0,4 \%$

$5,950,000 \times \mathrm{mm}^{3}$

$18,1 \mathrm{~g} / \mathrm{dl}$

$54,1 \%$

$91 \mathrm{fL}$

$14,2 \%$

$247,000 \times \mathrm{mm}^{3}$
Tabla 3. Resultados de los gases arteriales.

$\mathrm{pH}$

Pco2

Po2

$\mathrm{Na}+$

$\mathrm{K}+$

HCO3-

Hct
7,41

7,41
$30 \mathrm{mmHg}$
$54 \mathrm{mmHg}$
$136 \mathrm{mEq} / \mathrm{L}$
$3.0 \mathrm{mEq} / \mathrm{L}$
$19.0 \mathrm{mmol} / \mathrm{L}$
$58 \%$


Tabla 4. Resultado de otros laboratorios.

\begin{tabular}{lc}
\hline PT & $15,6 \mathrm{seg}$. \\
PTT & $30,7 \mathrm{seg}$. \\
Glicemia & $82 \mathrm{mg} / \mathrm{dl}$ \\
BUN & $8,6 \mathrm{mg} / \mathrm{dl}$ \\
Creatinina & $0,59 \mathrm{mg} / \mathrm{dl}$ \\
Proteína C reactiva & $0,63 \mathrm{mg} / \mathrm{dl}$ \\
\hline
\end{tabular}

\section{DISCUSIÓN}

La cardiopatía congénita más común es la comunicación interauricular, corresponde al 30\% a $40 \%$ de las cardiopatías congénitas en los adultos, con una prevalencia entre el 2,8 al 8,8 por 1.000 nacimientos. Se clasifica de acuerdo a su tamaño en: pequeña si es menor de 20 $\mathrm{mm}$ o grande si es mayor de $20 \mathrm{~mm}$. Suele localizarse en: 1) el ostium secundum, en la porción medial del septum auricular en la fosa oval; 2) ostium primum, en la porción anteroinferior del septum; y en 3) el seno venoso en la región posterior de la unión con la vena cava $^{(1)}$.

Las cardiopatías congénitas suelen ser detectadas a edades tempranas, pero muchos casos no son descubiertos hasta la edad adulta. Durante la primera década estos pacientes rara vez desarrollan hipertensión pulmonar pero es común después de la quinta década de la vida. En el estudio de Rui Providência et al.(1), los pacientes con hipertensión pulmonar fueron predominantemente adultos con una edad media de 52+ / -16 con un gran defecto $(19,7+/-9,6 \mathrm{~mm})$. Se cree que su ocurrencia durante la niñez se asocia a una hipertensión pulmonar idiopática coexistente con el defecto(1).

En el registro del Euro Heart Survey sobre pacientes adultos con enfermedades cardíacas congénitas, se encontró 1877 pacientes con comunicación interauricular, comunicación interventricular o defectos cianóticos con otra patología de base, 28\% tenían hipertensión pulmonar y $12 \%$ tenían síndrome de Eisenmenger ${ }^{(10)}$. En otro estudio más reciente, la prevalencia de la hipertensión arterial pulmonar entre 1824 adultos con cardiopatía congénita fue de 6,1\% y el 3,5\% tenía síndrome de Eisenmenger ${ }^{(10)}$. Basados en otros estudios como los de Rui Providência et al.(1) 77 pacientes $(42,5 \%)$ tuvieron una moderada a severa ampliación de la cámara cardíaca derecha y $48(26,5 \%)$ tuvieron hipertensión pulmonar, definido como PASP mayor de 40 $\mathrm{mmHg}$ (equivalente a una presión arterial pulmonar de $25-30 \mathrm{mmHg})$, y solo en $6(3,3 \%)$ fue diagnosticado el síndrome de Eisenmenger, con un alto predominio en la edad adulta $(64,0+/-7,9)^{(1)}$.

Se calcula que 3,2 millones de niños en todo el mundo que tengan una comunicación interauricular, comunicación interventricular o un ductus arterioso persistente aislado que no haya sido tratado durante la infancia, desarrollará una enfermedad vascular pulmonar con una morbilidad incapacitante de hipoxemia asociada a shuts invertidos sumado a una corta expectativa de vida(2).

Hay tres millones de niños en todo el mundo en riesgo de desarrollar una enfermedad vascular pulmonar asociada a una cardiopatía congénita. Se ha estimado que cerca de 600.000 nacimientos anuales que cursan con una cardiopatía congénita significativa, el 50\% fallece por infecciones o por falla cardíaca durante la infancia (2). Esta es una enfermedad que puede ser curada con una pronta intervención quirúrgica (Antes de los dos años). A nivel mundial solo del $2 \%$ al $15 \%$ de los pacientes con un significativo shut intra o extracardíaco logran recibir una intervención curativa $^{(2-6)}$.

Para desarrollar una enfermedad vascular pulmonar se ve implicada la hipertensión pulmonar, la cual según Simonneau et al. ${ }^{(8)}$ se clasifica de acuerdo a su causa en los siguientes grupos: 1) hipertensión arterial pulmonar 
idiopática familiar; 2) hipertensión venosa pulmonar; 3) hipertensión pulmonar asociada con hipoxemia; 4) hipertensión pulmonar asociado a una enfermedad trombótica crónica, enfermedad embolica o ambas y 5) misceláneas ${ }^{(7,8)}$; por lo tanto, de acuerdo al caso, este paciente pertenece al grupo uno ${ }^{(2)}$. También clínicamente se pueden clasificar las enfermedades cardíacas congénitas en: 1) síndrome de Eisenmenger; 2) moderado a gran Shut con hipertensión pulmonar severa pero todavía no invertido (no cianosis); 3) pequeños defectos con hipertensión arterial pulmonar (cuadro clínico similar al de la hipertensión arterial pulmonar idiopática) y 4) hipertensión arterial pulmonar después de reparar la enfermedad cardíaca congénita (persistente o recurrente) $)^{(10)}$.

Fisiopatológicamente, la causa más aceptada se basa en un incremento del flujo sanguíneo pulmonar y en la presión cuña de la pulmonar provocando un remodelamiento vascular desfavorable. Se genera una disfunción de las células endoteliales, desequilibrio en los mediadores vasoactivos, incluidos la prostaciclina, tromboxanos, endotelina, óxido nítrico, GMP cíclico, factor de crecimiento transformante beta 1 , factor de crecimiento del endotelio vascular, y la del factor de crecimiento de fibroblastos, llevando a vasoconstricción, inflamación, trombosis, proliferación celular, alteración de la apoptosis y la fibrosis( ${ }^{(2)}$.

Todos estos factores (cardiopatía congénita y enfermedad vascular pulmonar) más la no oportuna corrección del defecto congénito causa la formación del síndrome de Eisenmenger; el cual fue descrito por primera vez por Víctor Eisenmenger ${ }^{(9)}$. Posteriormente, Paul Wood $^{(2,9)}$ fue quien lo definió como una hipertensión pulmonar a nivel sistémico, asociada a un alto índice de resistencia vascular pulmonar (PVRI) (> $800 \mathrm{~s} / \mathrm{cm}^{5}$ o $10 \mathrm{WU} \mathrm{m}^{2}$ ) con un Shut invertido o bidireccional ${ }^{(2)}$.

De acuerdo con las guías para el manejo de enfermedades congénitas se define como una enfermedad cardíaca congénita inicial- mente con un Shut a nivel pulmonar porque induce a su vez una enfermedad vascular pulmonar severa e hipertensión arterial pulmonar, resultando un Shut de dirección invertida y una cianosis central. La supervivencia de estos pacientes, se aproxima a la tercera y hasta la cuarta década pero hay casos que han llegado hasta la séptima década de la vida. Los principales factores que influyen en un mal pronóstico para este tipo de pacientes son la falla cardíaca derecha y el deterioro progresivo de la capacidad de ejercicio.

Las características clínicas de esta patología son cianosis central, disnea, fatiga, hemoptisis, síncope, cefalea, mareo y palpitaciones principalmente; y como complicaciones se pueden encontrar también hemoptisis, ACV, abscesos cerebrales, secundarios a eritrocitosis, colelitiasis, disfunción renal, hiperuricemia, anormalidades en la coagulación, en particular, la trombosis parietal de arterias pulmonares proximales ampliada que se puede encontrar hasta en un $20 \%$ de los pacientes y puede causar embolización periférica e infartos pulmonares asociados a disfunción biventricular y reducción de la velocidad del flujo pulmonar ${ }^{(9,10)}$.

La prueba de reactividad vascular pulmonar aclara si la hipertensión pulmonar es reversible o irreversible usando óxido nítrico inhalado, epoprostenol, adenosina, nitroglicerina, nitroprusiato entre otros. El tratamiento, ideal sería la intervención quirúrgica teniendo en cuenta ciertas recomendaciones como son niños con un gran Shut de izquierda a derecha o evidencia de una elevada resistencia vascular pulmonar donde se debe cerrar el defecto durante los primeros doce a dieciocho meses de vida. Pacientes con predisposición a la enfermedad vascular pulmonar (trisomía 21), a quienes se recomienda reparar en los seis primeros meses de edad. En el tronco arterioso o transposición de grandes arterias con una comunicación interventricular, debe ser reparada en los primeros meses de vida. 
El tratamiento de tipo farmacológico ideal se basa en el uso de inhibidores de la endotelina, inhibidores de la fosfodiesterasa 5 o con inhibidores de los prostanoides y aunque se asocian a costos muy elevados, el medicamento más económico y de fácil acceso es el inhibidor de la fosfodiesterasa 5: el Sildenafil.

Como última opción, el trasplante de pulmón con reparación del defecto cardíaco o un trasplante de corazón y pulmón se deben considerar si hay disminución del estado funcional, episodios de insuficiencia cardíaca derecha, y un avanzado deterioro de cambios hemodinámicos, en especial un pobre funcionamiento ventricular derecho y elevados niveles de Péptido Natriurético Auricular tipo $\mathrm{B}^{(10)}$.

\section{CONCLUSIÓN}

Las cardiopatías congénitas actualmente están en aumento pero pueden ser detectadas e intervenirse quirúrgicamente a tiempo, idealmente antes de los dos años de edad, pero aún así, cada año solo una mínima parte recibe un tratamiento adecuado. Se debe garantizar una óptima calidad de vida.

\section{REFERENCIAS}

1. Rui Providência et al. Hipertensão pulmonar emdoentescomcomunicação interauricular ostium secundum - haverárelaçãocom a complexidade ecocardiográfica. Rev Port Cardiol 2009; 28:1087-1096.

2. Ian Adatia, Shyam S. Kothari, Jeffrey A. Feinstein. Pulmonary Hypertension Associated With Congenital Heart Disease: Pulmonary Vascular Disease: The Global Perspective. (CAmerican College of Chest Physicians. Chest 2010;52S-61S.

3. Ángel Sánchez-Recalde, José M. Oliver, Guillermo Galeote, Ana González, Luis Calvo, Santiago Jiménez-Valero, Raúl Moreno, and José L. López-Sendón. Atrial Septal Defect With Severe Pulmonary Hypertension in Elderly Patients: Usefulness of Transient Balloon Occlusion. Rev Esp Cardiol 2010;63(7):860-864.
4. Diaconu Camelia Cristina. Atrial Septal Defect in an Elderly Woman a Case Report. (C) 2011, Carol Davila University Foundation. Journal of Medicine and Life January-March 2011; 4(1):91-93.

5. Min Goo Lee, Jum Suk Ko, Hyun Ju Yoon, Kye Hun Kim,YoungkeunAhn, Myung Ho Jeong, JeongGwan Cho, Jung Chaee Kang and Jong Chun Park. An Unusual Presentation of an Atrial SeptalDefect. Copyright (C) 2009 Korean Society of Echocardiography. J Cardiovasc Ultrasound 2009;17(4):151-152.

6. Juan Lacalzada, Cristina Enjuanes, Maria Manuela Izquierdo, Antonio Barragán Acea, Alejandro De La Rosa, and Ignacio Laynez. Pulmonary Valve Infective Endocarditis in an Adult Patient with Severe Congenital Pulmonary Stenosis and Ostium Secundum Atrial Septal Defect. Copyright (C) 2010 Juan Lacalzada et al.Cardiology Research and Practice Volume 2010, Article ID 798956, p. 1-3.

7. Harrison W. Farber, MD, Joseph Loscalzo, MD, Ph.D. Pulmonary Arterial Hypertension. Copyright (C) 2004 Massachusetts Medical Society. N Engl J Med 2004;351:1655-1665.

8. Gerald Simonneau, MD, Nazzareno Galié MD, Lewis J. Rubin, MD, David Langleben, MD, Werner Seeger, MD, Guido Domenighetti, MD, Simon Gibbs, MD, Didier Lebrec, MD, Rudolf Speich, MD, Maurice Beghetti, MD, Stuart Rich, MD, Alfred Fishman, MD. Clinical Classification of Pulmonary Hypertension. (C) 2004 by the American College of Cardiology Foundation. JACC 43(12) Suppl S. June 16, 2004:5S-12S.

9. Florencio B. Garofalo. El síndrome de Eisenmenger en el adulto. (C) 1994-2003 CETIFACBioingeniería UNER. Rev Fed Arg Cardiol 2003;32:84-92.

10. Helmut Baumgartner, Philipp Bonhoeffer, Natasja M.S. De Groot, Fokko de Haan, John Erik Deanfield, Nazzareno Galie, Michael A. Gatzoulis, Christa Gohlke-Baerwolf, Harald Kaemmerer, Philip Kilner, Folkert Meijboom, Barbara J.M. Mulder, Erwin Oechslin, Jose M. Oliver, Alain Serraf, Andras Szatmari, Erik Thaulow, Pascal R. Vouhe, Edmond Walma. European Society of Cardiology Guidelines for the management of grown-up congenital heart disease (new version 2010). (OThe European Society of Cardiology 2010. European Heart Journal 2010;31:2923-2924 y 2949-2950. 
Universidad

Surcolombiana

\title{
OFERTAS ACADÉMICAS FACULTAD DE SALUD UNIVERSIDAD SURCOLOMBIANA
}

PREGRADO

\section{PROGRAMA DE MEDICINA}

\author{
Registro SNIES: 338 \\ Jornada: \\ Diurna \\ Duración: $\quad 12$ semestres \\ Metodología: Presencial \\ Sede: Neiva \\ Título a Otorgar: Médico (a) \\ Acto por el cual se constituyo el Programa:
}

Acuerdo No. 100 de 1982

Acuerdo de Acreditación: Resolución No. 6455 del 23 de julio de 2010

Ministerio de Educación Nacional

Año de Iniciación de actividades Docentes

1982

\section{PROGRAMA DE ENFERMERÍA}

Acreditación de alta calidad:

Resolución 7444 del Ministerio de Educación Nacional de 30 noviembre de 2007

Jornada: Diurna

Duración: $\quad 10$ semestres

Metodología: Presencial

Sede: Neiva

Título que otorga: Enfermera - Enfermero

Información

Email:enfermeria@usco.edu.co

Dirección: Calle 9 con Carrera 14, contiguo al Hospital Universitario Hernando Moncaleano Perdomo 